Startup challenge:

Breast cancer

competition aims to

spur new companies

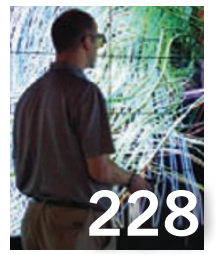

3D labs:

Virtual reality adds

a new dimension to

biomedical research

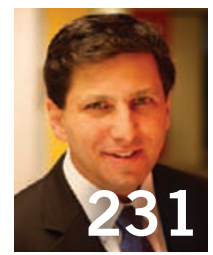

Social anxiety:

Social media use

threatens to unblind

ongoing clinical trials

\title{
As drug target reemerges, the question is to block or stimulate it
}

More than two decades ago, drugmakers searching for new hypertension medications unearthed a mysterious new cell receptor that responded to a hormone known as angiotensin II. This peptide hormone constricts blood vessels, but, oddly, blocking the so-called angiotensin II receptor type 2 (AT2) appeared to have no effect on blood pressure, so the target was largely ignored by drug developers. "Big pharma really just left the AT2 receptor by the side of the road," says Tom McCarthy, chief executive of Spinifex Pharmaceuticals, a company based in Melbourne, Australia, that is exploring the promise of targeting AT2.

Fast forward to today, and scientists now know that AT2 plays a part in everything from tissue repair to inflammation to pain response. A handful of companies are hustling to develop compounds that either block or stimulate this receptor to treat inflammatory diseases, nerve injuries, hypertension and more. In a paper published last month, researchers from Spinifex and their collaborators reported the first clinical data on a compound that binds to AT2 called EMA401. Results from the placebo-controlled phase 2 trial suggest that EMA401, which blocks the receptor, can blunt lingering nerve pain due to damage caused by the shingles virus ${ }^{1}$.

AT2 is just one of two receptors known to bind angiotensin II. Several medications that block the other receptor, AT1, have already received market approval for hypertension, diabetic nephropathy and congestive heart failure. When AT2 was first discovered, researchers thought the receptor was "just a little brother," says Thomas Unger, scientific director of CARIM, Maastricht University's School for Cardiovascular Diseases in the Netherlands. But Unger and his colleagues now know that AT2 has a "very peculiar and unique combination of effects, which is completely different from the AT1 receptor."

The phase 2 data recently reported by Spinifex came from a trial involving 183 people who had suffered from burning pain and hypersensitivity due to postherpetic neuralgia, a complication of shingles, for at least six months. The researchers randomly assigned half the participants to receive 100 milligrams of EMA401 twice a day for 28 days; the other half received a placebo.

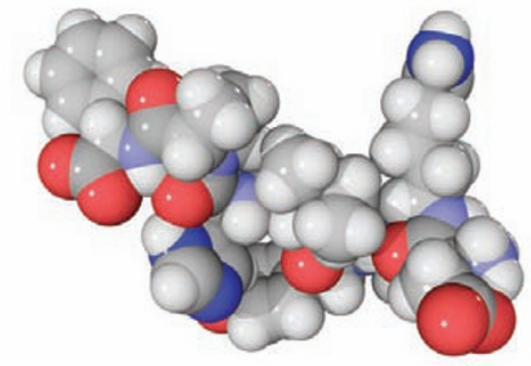

Blocked out: The hormone angiotensin II.

Those given EMA401 reported significantly less pain than those on placebo. By the last week of the study, about a third of the participants who took EMA401 experienced a 50\% reduction in pain; by comparison, only $18 \%$ in the placebo group achieved that level of relief.

A paper ${ }^{2}$ published in 2013 suggests a possible mechanism for the difference in pain scores: EMA401 inhibits pain responses in human and rat sensory neurons, the same neurons that respond to capsaicin, the compound that gives chili peppers their heat. Spinifex hopes to launch another, longer study in the coming months to examine whether raising the dose could increase the benefit.

\section{A stimulating difference}

Whereas Spinifex has focused on blocking the AT2 receptor, other developers are trying to stimulate it with AT2 agonists. The number of AT2 receptors increases after ttraumas such as heart attack or stroke in the damaged tissue, and the receptor appears to have a role in tissue repair.

For example, Lanthio Pharma, a Netherlandsbased company, published a paper last year showing that its AT2 agonist, LP2-3, provides some protection against lung injury in a mouse model of neonatal chronic lung disease ${ }^{3}$. And Vicore Pharma, a drug company based in Gothenburg, Sweden, demonstrated that its agonist, called Compound 21 (C21), can improve recovery in rats following spinal cord injury ${ }^{4}$.

As a first step, however, Vicore will probably focus on pulmonary fibrosis, a disease in which the lungs become increasingly scarred. "We're a small company," says Per Jansson, managing director of Vicore. "That limits us." Because pulmonary fibrosis is such a serious disease, the trials will probably be shorter and less costly. Vicore is collaborating with University College London to conduct a phase 1 trial that is slated to begin this fall.

Meanwhile, some researchers have revisited the idea that the AT2 receptor can be targeted to treat hypertension. The Japanese company Mitsubishi Tanabe Pharma, based in Osaka, has developed an agonist called MP-157. This compound is currently being tested in a phase 1 trial in Europe for hypertension.

Whether an AT2 agonist could have any impact on hypertension remains to be seen, though. Unger's research shows that AT2 agonists like C21 can cause dilation of blood vessels, but that effect doesn't seem to translate into lower blood pressure ${ }^{5}$. However, Lucinda Hilliard, a researcher at Monash University near Melbourne, points out that all the research on AT2 and hypertension has been done in male animals. "Females have a lot more of the AT2 receptor," she says. So Hilliard and her colleagues are investigating in preclinical studies whether females will respond differently. And although AT2 agonists may not lower blood pressure on their own, they do seem to have an effect when AT1 is blocked. So it's possible they could work well in concert with other hypertension drugs, she says.

AT2 is "still a mysterious receptor, sometimes exerting beneficial effects and sometimes exerting bad effects," says Jan Danser, a pharmacologist who studies AT2 at the Erasmus University Medical Center in Rotterdam, the Netherlands. "That makes it very complicated as a drug target," he adds. "We don't even know whether we should block it or stimulate it."

Cassandra Willyard

1. Rice, A.S.C. et al. Lancet doi:10.1016/S01406736(13)62337-5 (5 February 2014).

2. Anand, U. et al. Eur. J. Pain 17, 1012-1026 (2013).

3. Wagenaar, G.T. et al. Am J. Physiol. Lung Cell Mol. Physiol. 305, L341-L351 (2013).

4. Namsolleck, P. et al. Neurobiol. Dis. 51, 177-191 (2013).

5. Foulquier, S., Steckelings, U.M. \& Unger, T. Curr. Hypertens. Rep. 14, 403-409 (2012). 